ARTICLE

\title{
FtsZ-independent septal recruitment and function of cell wall remodelling enzymes in chlamydial pathogens
}

\author{
Antonio Frandi ${ }^{1}$, Nicolas Jacquier ${ }^{2}$, Laurence Théraulaz ${ }^{1}$, Gilbert Greub ${ }^{2}$ \& Patrick H. Viollier ${ }^{1}$
}

\begin{abstract}
The nature and assembly of the chlamydial division septum is poorly defined due to the paucity of a detectable peptidoglycan (PG)-based cell wall, the inhibition of constriction by penicillin and the presence of coding sequences for cell wall precursor and remodelling enzymes in the reduced chlamydial (pan-)genome. Here we show that the chlamydial amidase (AmiA) is active and remodels PG in Escherichia coli. Moreover, forward genetics using an $E$. coli amidase mutant as entry point reveals that the chlamydial LysM-domain protein NIpD is active in an $E$. coli reporter strain for PG endopeptidase activity $(\Delta n / p l)$. Immunolocalization unveils NIpD as the first septal (cell-wall-binding) protein in Chlamydiae and we show that its septal sequestration depends on prior cell wall synthesis. Since AmiA assembles into peripheral clusters, trimming of a PG-like polymer or precursors occurs throughout the chlamydial envelope, while NIpD targets PG-like peptide crosslinks at the chlamydial septum during constriction.
\end{abstract}

\footnotetext{
${ }^{1}$ Department of Microbiology and Molecular Medicine, Institute of Genetics and Genomics in Geneva (iGE3), Faculty of Medicine/CMU, University of Geneva, Rue Michel Servet 1, 1211 Genève 4, Switzerland. ${ }^{2}$ Center for Research on Intracellular Bacteria (CRIB), Institute of Microbiology, University Hospital Center and University of Lausanne, Bugnon 48, 1011 Lausanne, Switzerland. Correspondence and requests for materials should be addressed to P.H.V. (email: patrick.viollier@unige.ch).
} 
T he stress-bearing peptidoglycan (PG)-based cell wall protects bacterial cells from physical and chemical insults. PG (also known as murein) synthesis occurs throughout the envelope and at the division septum ${ }^{1,2}$. The septal PG along with constriction force by the cytokinetic ring assembled from FtsZ tubulin directs the envelope into an annular structure at the division plane to enable membrane fusion, ultimately compartmentalizing the dividing cell into two separate daughter chambers (Fig. 1a) ${ }^{3,4}$. The building block of PG is lipid II, an $N$-acetyl-glucosamine(GlcNAc)- $N$-acetyl-muramic acid (MurNAc)-pentapeptide unit carried by the phosphorylated isoprenoid bactoprenol (C55 P). During PG synthesis, the MurNAc-GlcNAc disaccharide units are polymerized into linear glycan strands by transglycosylase enzymes. The growing polymer is further fortified by crosslinking of the pentapeptide moieties by transpeptidases known as penicillin-binding proteins (PBPs; Fig. 1b). Upon synthesis of the septal PG and the ensuing compartmentalization, the septal PG is split to accommodate daughter cell separation, a task executed by PG remodelling enzymes such as lytic transglycosylases, amidases ( $N$-acetylmuramoyl- $L$-alanine hydrolases) and peptidases (LD-carboxypeptidases and DD-endopeptidases) that act on the glycan, amide or peptide bonds in PG, respectively (Fig. 1a,b) ${ }^{3}$.

In addition to providing constriction force, FtsZ organizes septal PG synthesis and remodelling events ${ }^{1,2}$. Although most bacteria rely on FtsZ for division, several bacterial lineages, such as pathogens belonging to the phylum Chlamydiae, do not encode primary structural homologues of FtsZ in their genomes ${ }^{1}$. Thus, alternative organizers of PG synthesis/remodelling and of cytokinesis must exist. In principle, owing to their obligate intracellular life style and a protective network of proteins with disulphide bridges on the surface of elementary bodies (the infectious extracellular developmental stage), Chlamydiae should not need PG for protection from osmotic stress. Interestingly, however, despite the massive reduction in coding capacity of chlamydial genomes, a seemingly functional lipid II biosynthetic pathway along with several putative PG biosynthetic (transpeptidases, for example, FtsI) and predicted remodelling enzymes (putative amidases and endopeptidases) are encoded ${ }^{5-8}$. The latter suggests that chlamydial pathogens polymerize a septal PG derivative (or at least a PG remnant). Indeed, immunofluorescence labelling with antibodies to the Ribi adjuvant that contains mycobacterial cell wall skeleton or direct fluorescent labelling of a modified D-amino acid dipeptide revealed a non-proteinaceous PG-like substance or at least a dipeptide-derived PG precursor at the septum ${ }^{9,10}$. Moreover, penicillins (inhibitors of PBPs) block chlamydial division ${ }^{8,10,11}$. However, no coding sequences for known PG transglycosylation enzyme homologues are found in the genomes of chlamydial pathogens ${ }^{8}$, raising the intriguing possibility that this PG-like material lacks chains of glycan polymers and that instead the disaccharide units from lipid II remain unpolymerized after transpeptidation. Alternatively, unknown transglycosylation enzymes may promote glycan chain formation within the chlamydial cell wall. Modification of PG-like material, its synthesis in reduced amounts and/or its confinement in space or time could reflect an adaptation of chlamydial pathogens to the host by reducing the activation of NOD1/2 intracellular pattern recognition receptors that detect MurNAc-containing muropeptide fragments ${ }^{12}$.

Division in the absence of classical PG and FtsZ as that seen for the L-form bacteria and mycoplasmas belonging to the phylum Firmicutes occurs in an erratic and inefficient manner by membrane blebbing, budding or stretching ${ }^{1,13,14}$. By contrast, cell division in the phylum Chlamydiae is highly coordinated and regular, resembling the binary fission of cocci ${ }^{8,15}$. It is unknown how chlamydia execute division and if they remodel their septal PG-like material, but recently the first septal proteins of Chlamydiae have been identified ${ }^{11}$. Escherichia coli mutants lacking all three amidase paralogues offer a convenient system to probe for septal PG remodelling by amidase-like activities, as inactivation of the three amidase genes (amiA, amiB and $a m i C)$ prevents cell separation, yielding a chaining phenotype ${ }^{16}$. Interestingly, the barrier function of the outer membrane (conferred by lipopolysaccharide ${ }^{17}$ (LPS), which also uses $\mathrm{C} 55 \sim \mathrm{P}$ as a carrier for the biosynthesis of its precursor; Fig. 1b) is compromised in the $\triangle a m i A, \triangle a m i B ; \triangle a m i C$ (henceforth $\triangle A B C$ ) triple mutant for reasons that are unclear ${ }^{18}$.

Here, using $E$. coli $\triangle A B C$ as a surrogate host, we first confirmed that chlamydial AmiA orthologues restore cell separation and LPS barrier function, indicating that they are indeed active amidases. We then isolated a suppressive mutation in the E. coli gene encoding the NlpI lipoprotein that restores LPS function and alters the PG peptide crosslinking ratio in $\triangle A B C$ cells. We provide evidence that chlamydial NlpD can bind PG in vitro and that it has PG peptidase activity in E. coli cells that are mutant for nlpI in vivo. Importantly, immunolocalization of dividing chlamydial (Waddlia chondrophila) cells unveils NlpD as the first septal cell-wall-binding protein and shows that it depends on a PG-like polymer for localization to the division septum. As AmiA is distributed in the cell envelope, our results support a model in which AmiA trims a PG-like polymer or lipid II throughout the envelope, while NlpD acts on peptide crosslinks at the division septum of human chlamydial pathogens.

\section{Results}

Activity and peripheral localization of chlamydial amidases. As chlamydial pathogens are typically small and difficult to grow, we exploited the robust growth and larger cell size of $W$. chondrophila, a member of the Chlamydiales order and a strict intracellular pathogen associated with bovine abortion and human miscarriage, for immunolocalization studies ${ }^{19,20}$. As it has so far not been possible to engineer targeted gene disruptions in W. chondrophila as for most members of the Chlamydiales, we complemented our cytological experiments with functional studies using E. coli as a surrogate host.

W. chondrophila AmiA $\left(\mathrm{AmiA}^{W c h}\right)$ is encoded in a gene cluster with predicted cell division and PG precursor (lipid II) biosynthesis enzymes (Fig. 1c). Akin to other chlamydial AmiA orthologues, AmiA ${ }^{W c h}$ exhibits $43 \%$ similarity (113/259) and $27 \%$ identity $(70 / 259)$ to E. coli AmiA ( $\mathrm{AmiA}^{E c o}$ ), and features the predicted catalytic residues within the LytC/Amidase_3 signature domain of amidases (Fig. 1d, Supplementary Fig. 1 and Supplementary Table 1). Interestingly, primary structure predictions suggest that chlamydial amidases lack the autoinhibitory alpha-helix (Supplementary Fig. 1) that occludes substrate access of the E. coli amidases and that must first be displaced by a cognate amidase activator for the acquisition of full enzymatic activity $^{21,22}$. This raises the possibility that the active site of the chlamydial amidase homologues could be in a constitutively open (active) state. $A m i A^{W c h}$ was able to rescue the cell separation defect (chaining phenotype) of the E. coli $\triangle A B C$ mutant (Fig. 2a and Supplementary Fig. 2A) akin to $A \mathrm{miA}^{E c o}$. However, a significant amount of cell debris ('ghosts'; arrowheads in Fig. 2a and Supplementary Fig. 2A) accumulated in the cultures expressing AmiA ${ }^{W c h}$, presumably reflecting lysed cells from ectopic (un-restrained) amidase activity of AmiA ${ }^{W c h}$ (Fig. 2a) that cannot be properly controlled by E. coli. In support of this, we observed by way of a LacZ-based lysis assay that AmiA ${ }^{W c h}$ liberated LacZ much more efficiently from cells compared with $\mathrm{AmiA}^{E c o}$ (Supplementary Fig. 2B). Cell 'ghosts' were not seen 

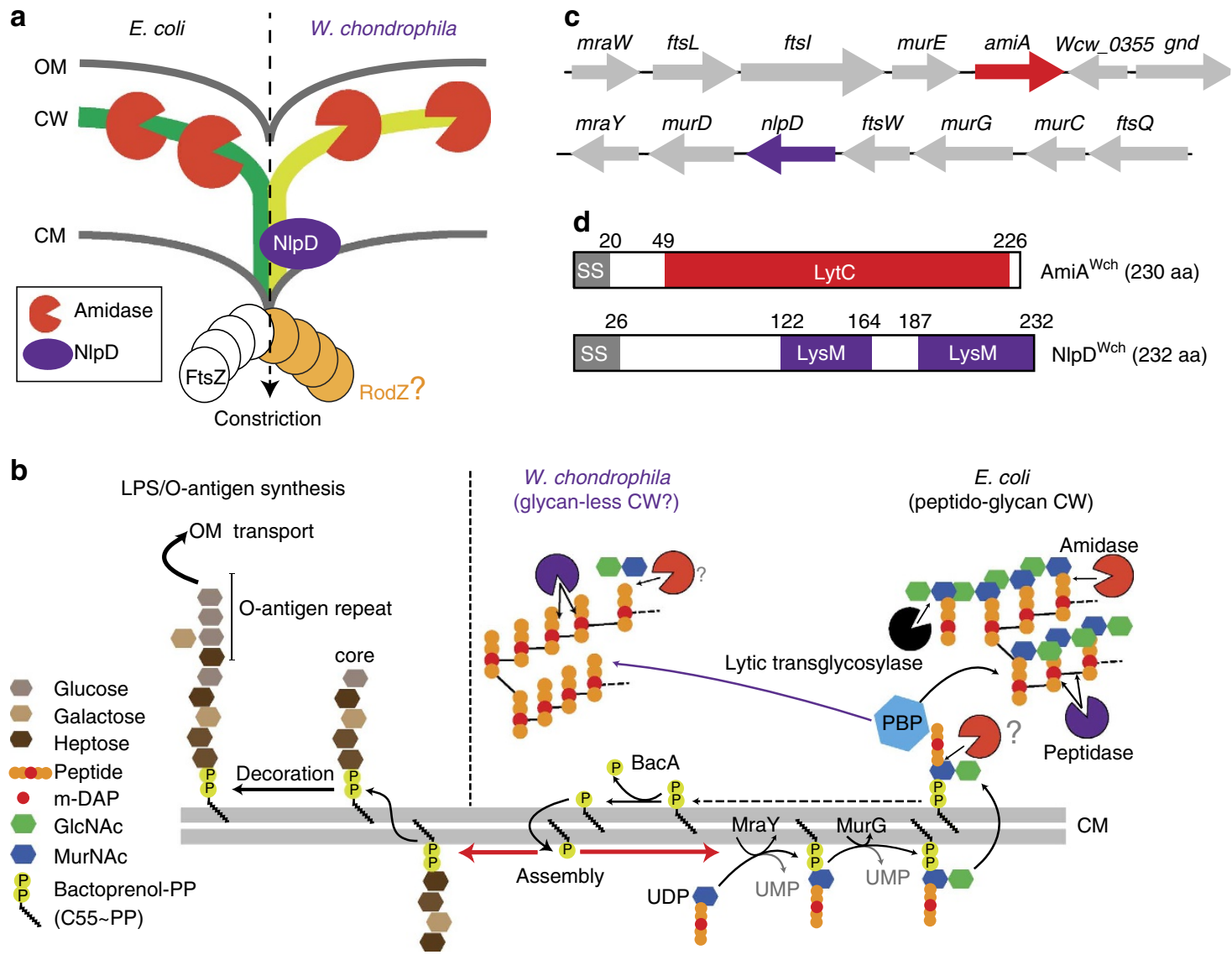

Figure 1 | Model of cell wall remodelling in dividing E. coli and W. chondrophila. (a) Coordinated envelope constriction in E. coli and W. chondrophila, a member of the Chlamydiales order, by the cytokinetic machinery and (putative) cell wall biosynthetic and remodelling enzymes including amidases and the putative endopeptidase NIpD from W. chondrophila ( $N / p D^{W c h}$ ). RodZ ${ }^{W c h}$ was recently identified as an early recruit to the division plane, raising the possibility that it acts in an early cytokinetic event ${ }^{11}$. (b) Schematic of cell wall assembly pathway in $E$. coli and the predicted pathway of a PG-like cell wall in $W$. chondrophila, as well as the O-antigen pathway for biosynthesis of LPS in the E. coli outer membrane (OM). Note that both pathways use bactoprenol $(C 55 \sim P)$ as membrane carrier for precursor assembly. The possible removal of the dissaccharide unit from PG or lipid II is indicated with a question mark and a transparent dissaccharide unit. (c) Gene organization of the region encompassing the coding sequences for AmiA and NIpD in W. chondrophila. Arrows indicate gene orientation in the W. chondrophila genome. (d) Predicted domain organization of W. chondrophila AmiA and NIpD. SS, signal sequence; LytC, (Pfam PF01520) amidase_3 domain; LysM, (Pfam PF01476) LysM-like PG-binding domain. Numbers refer to amino-acid positions in the translation product.

when AmiA ${ }^{W c h}$ derivatives with single, double and/or quadruple mutations in key catalytic residues (H55A, E70A, H124A and/or E194A; Supplementary Fig. 1) or AmiA ${ }^{\text {Eco }}$ were expressed (Fig. 2a), indicating that catalytic activity underlies the lysis phenotype. The catalytic mutants were also unable to support cell separation even though wild-type (WT) and most mutant $A_{m i A}{ }^{W c h}$ derivatives accumulated to comparable steady-state levels as determined by immunoblotting using polyclonal antibodies to $\mathrm{AmiA}^{W c h}$ (Supplementary Fig. 2C,D). Finally, to determine whether these functional characteristics are also retained in AmiA orthologues from other members of the Chlamydiales, we conducted complementation experiments with a plasmid expressing the AmiA orthologue from Simkania negevensis $\left(\mathrm{AmiA}^{\mathrm{Sne}}\right)$ or from Parachlamydia acanthamoebae $\left(\mathrm{AmiA}^{\text {Pac }}\right)^{23}$ (Supplementary Fig. 1) and found that both are also active as amidases, inducing lysis and supporting cell separation and ghost cell formation in E. coli (Supplementary Figs 2B,E,F and $3 \mathrm{~A}-\mathrm{C}$ ).

Further evidence that the chlamydial amidases indeed have lytic activity came from expression of WT and mutant AmiA ${ }^{W c h}$ in the Gram-negative Alpha-proteobacterium Caulobacter crescentus that naturally grows in hypo-osmotic fresh water niches $^{24}$ and is thus more prone to lysis when PG integrity is compromised. We observed that WT AmiA ${ }^{W c h}$, but not mutant derivatives, induced rapid lysis upon shifting aerated (shaking) C. crescentus cultures to stasis (Fig. 3a). Moreover, highperformance liquid chromatography (HPLC) analysis of muropeptides liberated from purified cell wall sacculi that had been digested with the $N$-acetyl-muramidase mutanolysin revealed that $\mathrm{AmiA}^{W c h}$ induces the appearance of several muropeptide fragments in $C$. crescentus that are not present in the control samples (from cells harbouring the empty vector), with a commensurate reduction in other muropeptide species (Fig. 3b).

As E. coli amidase mutants have compromised LPS-dependent outer membrane barrier function, they are unable to grow on medium containing detergents, including the bile acid deoxycholate in McConkey agar ${ }^{18,25}$ (Fig. 2b). Moreover, LPS is the receptor for bacteriophage $\varphi \mathrm{P} 1$ and the $\triangle A B C$ mutant displays an increased resistance towards $\varphi \mathrm{P} 1$ compared with $W T$ cells (Fig. 2c). Surprisingly, expression of WT AmiA ${ }^{W c h}$ corrected these deficiencies as well, while the AmiA ${ }^{W c h}$ catalytic mutants were unable or substantially reduced in their ability to support these functions (Fig. 2b,c). The $\triangle A B C$ mutant is also sensitive to 
a
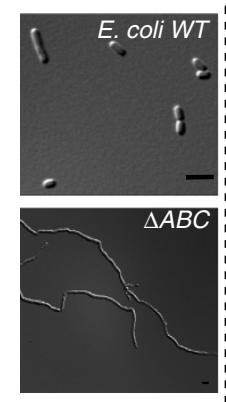

$A B C$
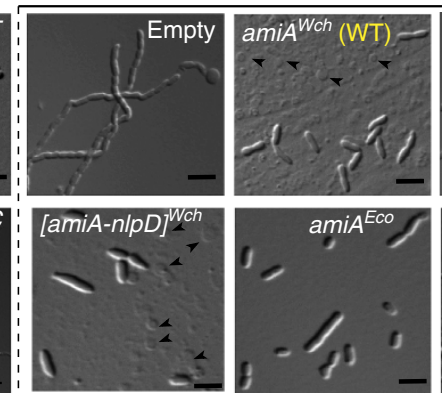

$\triangle A B C+\mathrm{P}_{\text {van }}-$ plasmid
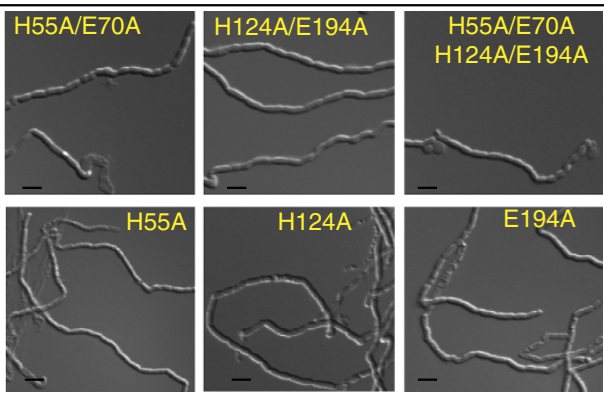

b
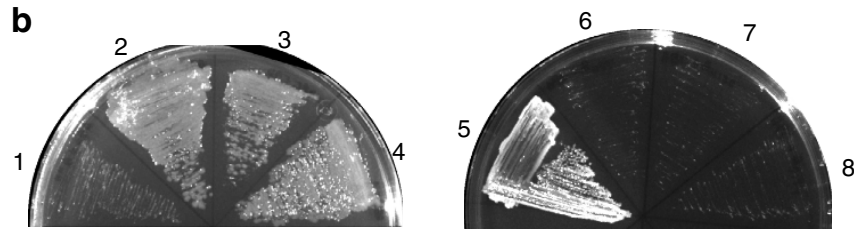

$\triangle A B C+\mathrm{P}_{\text {van }}-$ plasmid (1-8) on McConkey Agar + Glucose $(0.5 \%)$

\begin{tabular}{|c|c|c|c|}
\hline 1-empty & 2-amiA $A^{E c o}$ & 3-[amiA-nlpD] $]^{W c h}$ & 4-amiA ${ }^{W c h}$ \\
\hline $5-a m i A^{E c o}$ & 6-amiA ${ }^{W c h}(\mathrm{E} 194 \mathrm{~A})$ & 7-amiA ${ }^{W c h}(\mathrm{E} 124 \mathrm{~A})$ & 8-amiA ${ }^{W c h}(\mathrm{H} 55 \mathrm{~A})$ \\
\hline
\end{tabular}
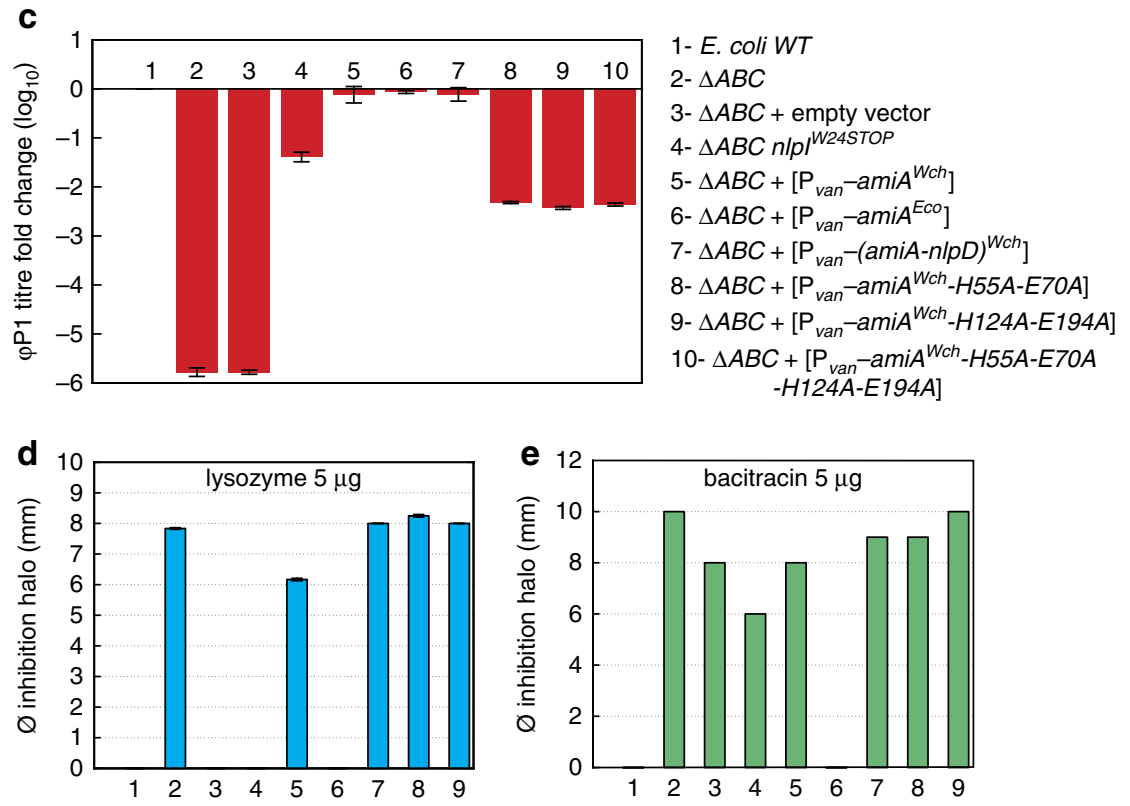

$$
\begin{gathered}
\text { 1- E. coli } W T \\
\begin{aligned}
& 4-\triangle A B C+ {\left[\mathrm{P}_{\text {van }}-(a m i A-n / p D)^{W c h}\right] } \\
& 7-\triangle A B C+ {\left[\mathrm{P}_{\text {van }}-a m i A^{W c h}\right.} \\
&\mathrm{H} 55 \mathrm{~A} \text { E70A }]
\end{aligned}
\end{gathered}
$$$$
\text { 2- } \triangle A B C
$$

$$
\begin{aligned}
& \text { 3- } \triangle A B C+ {\left[\mathrm{P}_{\text {van }}-a m i A^{W c h}\right] } \\
& 6-\triangle A B C+ {\left[\mathrm{P}_{\text {van }}-a m i A^{E c o}\right] } \\
& \text { 9- } \triangle A B C+ {\left[\mathrm{P}_{\text {van }}-a m i A^{W c h}\right.} \\
&\text { H55A E70A H124A E194A }]
\end{aligned}
$$

Figure 2 | W. chondrophila AmiA supports cell separation in E. coli $\triangle \boldsymbol{A B C}$ cells. (a) DIC images of $W T$ and $\triangle A B C ; \triangle A B C+\mathrm{P}_{\text {van }}-\mathrm{empty},-a m i A^{W c h},-[a m i A-$ $n / p D]^{W c h}$ and -amiA ${ }^{E c o}$. Arrowheads point to ghost cells; Scale bar, $4 \mu \mathrm{m}$. AmiA point mutants are highlighted in yellow. (b) Growth of WT and mutant E. coli strains on McConkey agar supplemented with $0.5 \%$ glucose (McCG). (c) $\varphi$ P1 titre in the indicated strains were calculated as described in Methods and is reported as the $\log _{10}$ fold change. Error bars show the s.d. Data are from three biological replicates. (d) Lysozyme sensitivity of the indicated E. coli strains. $0.25 \mathrm{ml}$ of saturated cultures were added to $5 \mathrm{ml}$ of LB top agar and plated, the indicated amount of lysozyme was then spotted on the cell overlay and incubated overnight at $30^{\circ} \mathrm{C}$. Differences in sensitivity are reported as the difference in size (diameter) of the inhibition halo (mm). Error bars show the s.d. Data are from three biological replicates. (e) Bacitracin sensitivity of the indicated E. coli strains. 250 microliters of saturated cultures were added to $5 \mathrm{ml}$ of LB top agar and plated, the indicated amount of bacitracin was then spotted on the cell overlay and incubated overnight at $30^{\circ} \mathrm{C}$. Differences in sensitivity are reported as the difference in size (diameter) of the inhibition halo (mm). Error bars show the s.d.

exogenously applied lysozyme (a muramidase) or bacitracin (an antibiotic interfering with $\mathrm{C} 55 \sim \mathrm{P}$ recycling through inhibition of the kinase BacA; Figs $1 \mathrm{~b}$ and $2 \mathrm{~d}, \mathrm{e})$. While expression of $\mathrm{AmiA}^{E c o}$ corrects all deficiencies of the $\triangle A B C$ mutant (Fig. 2a-f), $A m i A^{W c h}$ was unable to correct the bacitracin sensitivity of the $\triangle A B C$ mutant. We attribute this to the ectopic (lytic) activity of $A m i A^{W c h}$ that, in the absence of the autoinhibitory region that is found in amidases such as $\mathrm{AmiA}^{E c o}$ (Supplementary Fig. 1), leads to 'ghost' cell formation and an imbalance in PG precursors and/ or bactoprenol $(\mathrm{C} 55 \sim \mathrm{P})$ derivatives. Such an imbalance in 

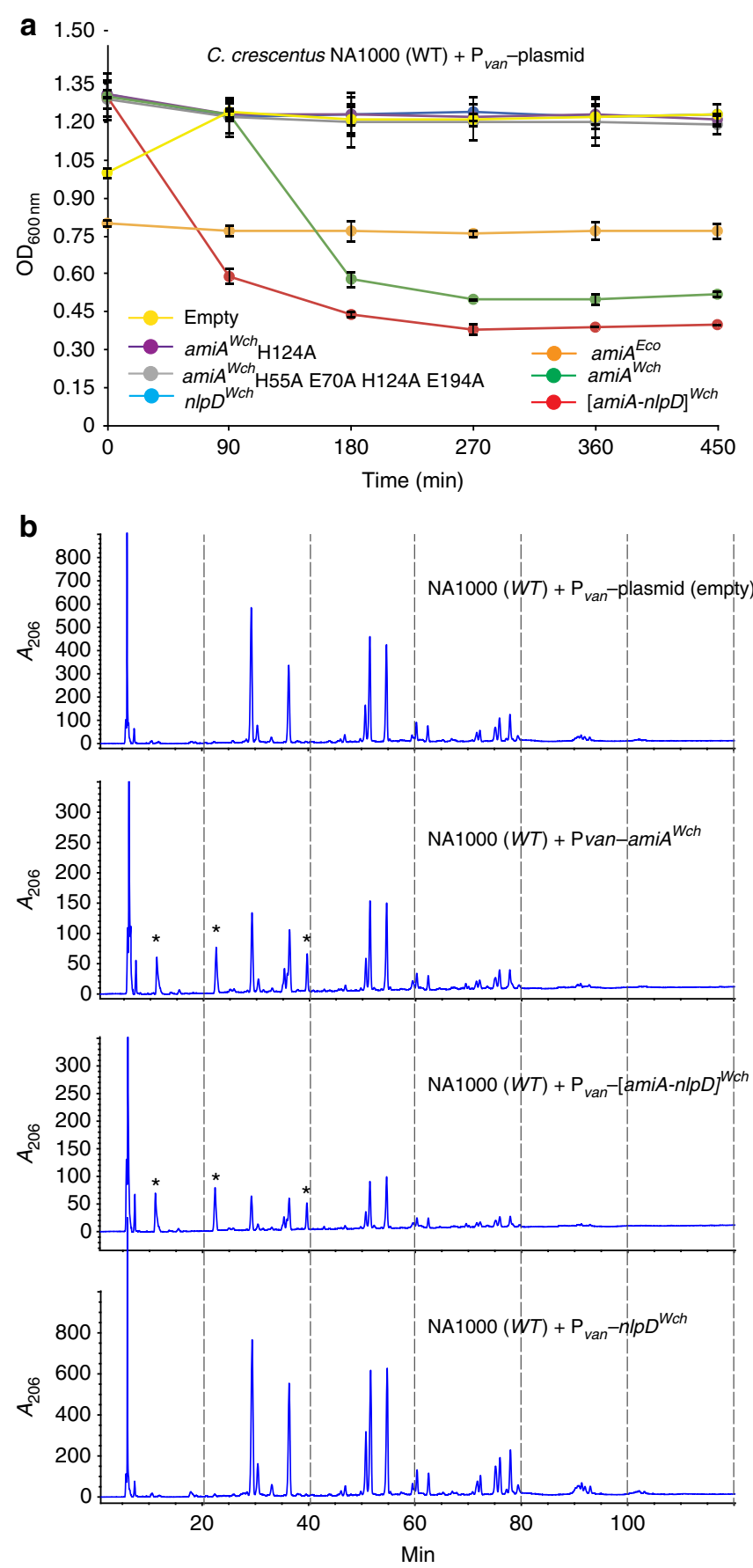

Figure 3 | Effect of AmiA ${ }^{\text {Wch }}$ derivatives on lysis of Caulobacter crescentus. (a) Overnight cultures of $C$. crescentus harbouring different constructs were left static at $30^{\circ} \mathrm{C}$ and $O_{600 \mathrm{~nm}}$ were recorded every 90 min. Lysis occurred in strains carrying $\left[\mathrm{P}_{\text {van }}-a m i A^{W c h}\right]$ and $\left[\mathrm{P}_{\text {van }}-(\operatorname{amiA}-n / p D)^{W c h}\right]$ while $\mathrm{OD}_{600 \mathrm{~nm}}$ of strains carrying $\left[\mathrm{P}_{\text {van }}-\right.$ $a_{\text {ami }} A^{W c h}$ H124A $],\left[P_{\text {van }}-a_{\text {ami }} A^{W c h}\right.$ H55A E70A H124A E194A $],\left[P_{\text {van }}-n / p D^{W c h}\right]$ and pMT335 were not affected. Error bars show the s.d. Data are from three biological replicates. (b) Muropeptide analysis of Caulobacter crescentus cell walls in the indicated strains harbouring different constructs as indicated on the figure. Differences among HPLC profiles are highlighted by asterisks.

C55 P might sensitize cells to inhibitors of the bactoprenol recycling pathway such as bacitracin. Taken together, we conclude that despite the massive genome reduction during the evolution of Chlamydiae, the coding sequence of a functional and lytic amidase has been retained.
To confirm that $A m i A^{W c h}$ is indeed expressed in dividing $W$. chondrophila cells, we measured the abundance of the amiA ${ }^{W c h}$ transcripts by reverse transcription quantitative (RT)-PCR postinfection (p.i.) of Vero host cells infected with $W$. chondrophila (Fig. 4a). We also raised polyclonal antibodies to $\mathrm{AmiA}^{\mathrm{Wch}}$ and probed for the presence of $A \mathrm{miA}^{\text {Wch }}$ by immunoblotting during growth of $W$. chondrophila (Supplementary Fig. 4). These experiments revealed the $a m i A^{W c h}$ transcript and the AmiA ${ }^{W c h}$ translation product to be detectable at all time points p.i. Moreover, we used the anti-AmiA ${ }^{W c h}$ antiserum for immunofluorescence microscopy of cells $24 \mathrm{~h}$ p.i. and observed AmiA ${ }^{W c h}$ in clusters in the cell envelope and occasionally at the septum in deeply constricted cells (Fig. 4b). While our functional and cytological analyses provide compelling evidence that the chlamydial amidases are functional, expressed and at the correct subcellular compartment to process a PG-like polymer (synthesized by the PBP transpeptidases, PBP2 and/or PBP3) $)^{5,6}$, we cannot rule out that AmiA ${ }^{W c h}$ acts directly on the PG building block lipid II, splicing off the MurNAc-GlcNAc disaccharide once lipid II is flipped onto the periplasmic face of the cytoplasmic membrane and polymerized by PBPs (Fig. 1b). In fact, the companion paper by Klöckner et al. ${ }^{26}$ demonstrates that Chlamydiae pneumoniae AmiA can cleave lipid II in vitro. We thus hypothesize that AmiA is constitutively active and can release the MurNAc-GlcNAc disaccharide unit from Lipid II and/ or from a septal/peripheral PG-like polymer, even in the absence of a topological amidase activator ${ }^{3,4,22}$. It is also conceivable that chlamydial AmiA orthologs are important for bactoprenol $(\mathrm{C} 55 \sim \mathrm{P})$ recycling, which could be limiting due to ongoing LPS (O-antigen) precursor biosynthesis as in E. $\operatorname{coli}^{17}$ (Fig. 1a).

Amidases and endopeptidases influence LPS barrier function. Since E. coli amidases are required for proper LPS-dependent barrier function, they could modulate signalling by Toll-like innate immune receptors that detect bacterial cell envelope components such as LPS. To investigate how AmiA ${ }^{W c h}$ promotes LPS-dependent barrier function in $E$. coli $\triangle A B C$ cells, we isolated a spontaneous $\triangle A B C$ suppressor mutant $\left(\triangle A B C n l p I^{W 24 S T O P}\right)$ that is able to grow on McConkey agar (supplemented with $0.5 \%$ Glucose; Fig. 5a). While this mutant exhibits near WT sensitivity to $\varphi \mathrm{P} 1$ (Fig. 2c), the defects in cell separation, lysozyme and bacitracin sensitivity were not mitigated (Figs $2 c, d$ and $5 b$ ). Thus, the LPS-dependent barrier function can be genetically uncoupled from cell separation.

Genome re-sequencing of this suppressor mutant disclosed a nonsense mutation of the tryptophan codon at position 24 (TGG $\rightarrow \mathrm{TAG}$ ) in the $n l p I$ gene, encoding a tetratricopeptiderepeat (TPR)-containing lipoprotein required for virulence and adhesion in neonatal meningitis E. coli ${ }^{27,28}$. In support of the notion that the $n l p I^{W 24 S T O P}$ mutation is a loss-of-function allele, expression of WT nlpI from a plasmid in the $\triangle A B C$ nlpI $I^{W 24 S T O P}$ quadruple mutant again conferred the growth defect on McConkey agar medium typical of $\triangle A B C$ cells (Fig. 5a). Conversely, deletion of nlpI $\left(\Delta n l p I:: \mathrm{cm}^{\mathrm{R}}\right)$ from $\triangle A B C$ cells enabled growth of the resulting $\triangle A B C \triangle n l p I$ quadruple mutant on McConkey agar (Fig. 5c).

How might NlpI act? Since $\triangle A B C$ nlpI $I^{W 24 S T O P}$ quadruple mutant cells are nearly as sensitive to bacitracin compared with the $\triangle A B C$ parent, it is unlikely that NlpI acts in the bactoprenol $(\mathrm{C} 55 \sim \mathrm{P})$ recycling pathway (Fig. 1b). Interestingly, $n l p I$ is known to interact genetically with spr, a gene encoding a DDendopeptidase that cleaves the peptide cross-bridges between $\mathrm{m}$-DAP and $\mathrm{D}$-alanine in $\mathrm{PG}^{29}$. Moreover, loss-of-function mutations in $n l p I$ or overproduction of the m-DAP-D-alanine DD-endopeptidase Pbp7 both suppress the conditional lethality 
a

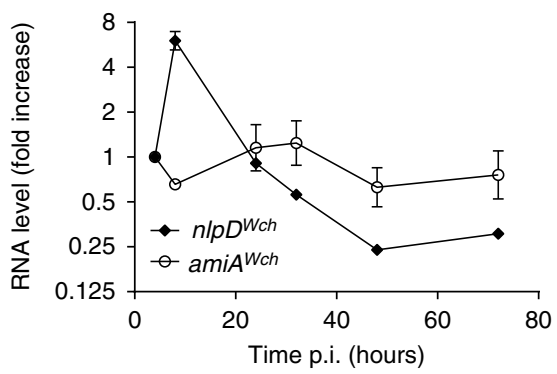

C

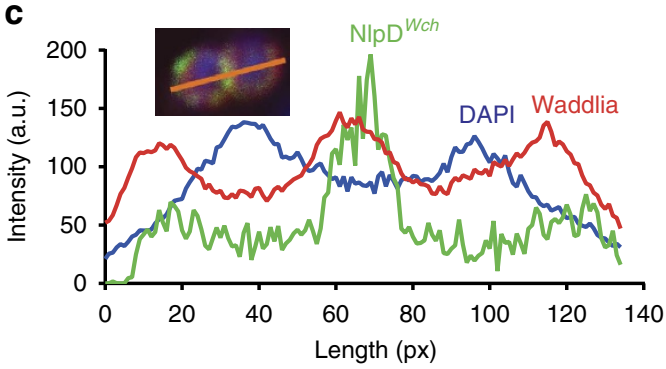

b

Immunofluorescence microscopy in W. chondrophila
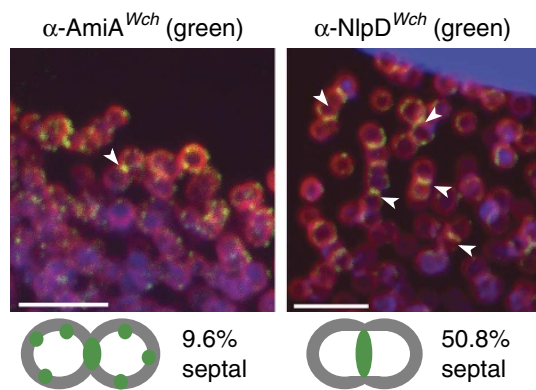

$\alpha-\mathrm{NIpD}^{W c h}$ (4h penicillin)

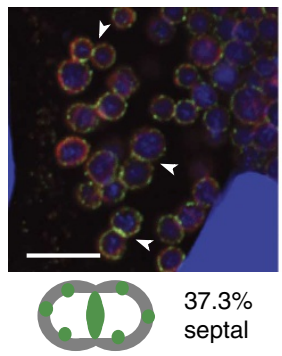

$\alpha-N I p D{ }^{W c h}$

(4h phosph.)

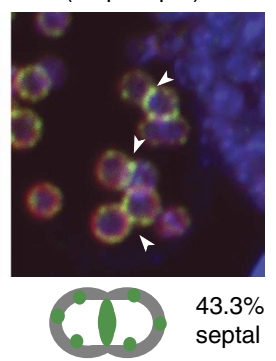

d Immunofluorescence microscopy in W. chondrophila
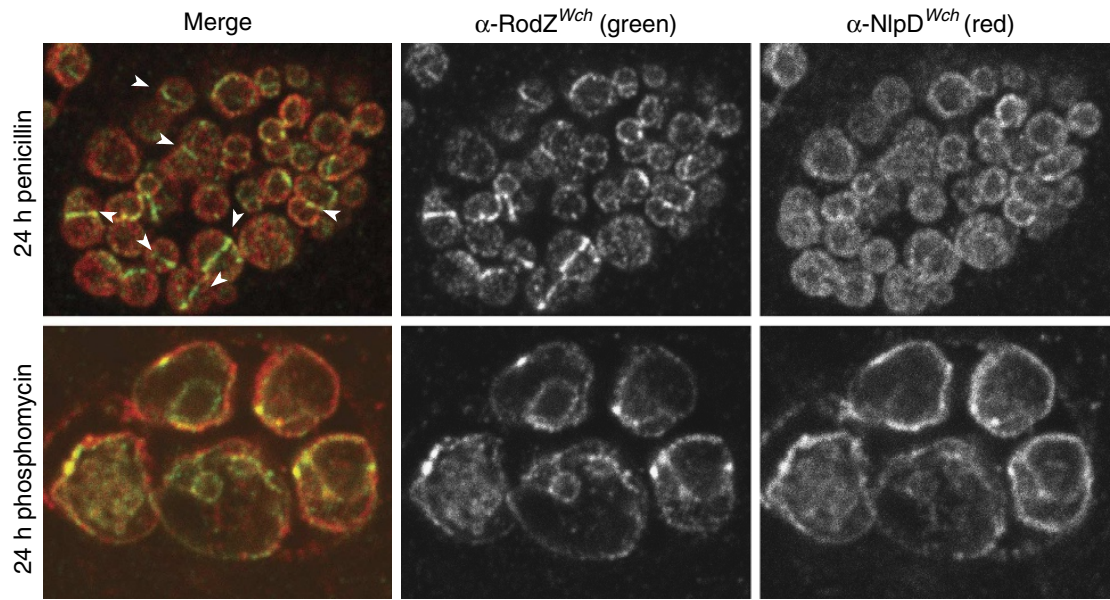

Figure 4 | NlpDWch localizes to the chlamydial division septum. (a) Transcript levels of $a m i A^{W c h}$ and $n l p D^{W c h}$ in Vero cells at different time points p.i. with W. chondrophila. Error bars show the s.d. Data are from three biological replicates. (b) NIpDWch localizes at the division plane (middle), while AmiA ${ }^{\text {Wch }}$ is localized at cell periphery with accumulation at constriction in dividing bacteria. Septal localization of NlpDWch is not affected by $4 \mathrm{~h}$ of penicillin or phosphomycin (phosph.) treatment $\left(500 \mu \mathrm{g} \mathrm{ml}^{-1}\right)$ administered $2 \mathrm{~h}$ p.i. Numbers indicate the fraction of cells with septal signal ( $\left.n=100\right)$. Shown are merged images of cells stained with DAPI (blue), anti-Waddlia antibodies (red) and antibodies (green) to either AmiA ${ }^{W c h}$ ( $\alpha-A m i A^{W c h}$ ) or NIpDWch $\left(\alpha-\mathrm{NlpD}^{W c h}\right)$. (c) Enrichment of NlpD Wch at the division plane is not due to overlapping cells. Z-stacks were observed by confocal immunofluorescence microscopy. Reconstruction of a vertical cut through the cells is depicted here (inset). Quantification of pixel ( $p x$ ) intensities using ImageJ confirmed a concentration of $\mathrm{NlpD}^{\text {Wch }}$ at the division plane (right). (d) Immunofluorescence micrographs using antibodies to NlpD Wch (red) and RodZWch (green) showing the delocalization of NlpD Wch after $24 \mathrm{~h}$ of penicillin and phosphomycin administered $2 \mathrm{~h}$ p.i. Note that the midcell localization of RodZ ${ }^{W c h}$ is maintained in the presence of penicillin, but not in the presence of phosphomycin as published previously ${ }^{11}$.

of an spr deletion $(\Delta s p r)$ mutant ${ }^{30,31}$. Consistent with the notion that inactivation of $n l p I$ affects and perhaps loosens septal PG, we observed that the poor growth of a $\Delta s p r$ strain on McConkey agar is attenuated by a $\Delta n l p I$ mutation (Fig. $5 \mathrm{~d}$ ), that $\Delta n l p I$ cells are sensitive towards increased expression of Spr or its paralogue YdhO (Fig. 6a) and that the inactivation of $n l p I$ in $W T$ or $\triangle A B C$ cells resulted in an increased muro-tetrapeptide monomer to dimer ratio as determined by HPLC analysis (Table 1; Supplementary Fig. 5A,B). On the basis of these results, we propose that NlpI (perhaps via the TPR repeat) negatively regulates Spr and other endopeptidases that convert murotetrapeptide dimers to monomers. Thus, a proper balance of amidase and DD-endopeptidase activities governs the barrier function of LPS.

Septal localization and cell wall remodelling by $\mathrm{NlpD}^{\mathrm{Wch}}$. Prompted by these functional interactions between the amidases 
a

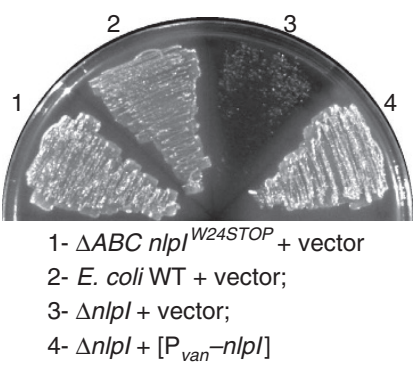

b

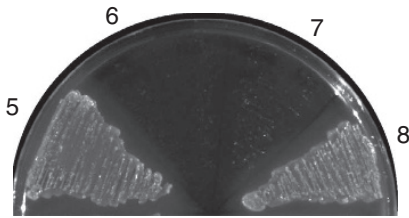

5- $\left.\Delta n / p l+\left[\mathrm{P}_{\text {van }}-n / p\right)\right]$

6- $\triangle A B C n / p l^{\text {W24STOP }}+\left[\mathrm{P}_{\text {van }}-n / p /\right]_{\mathrm{a}}$;

7- $\triangle A B C n / p l^{\text {W24STOP }}+\left[\mathrm{P}_{\text {van }}-n / p /\right]_{\mathrm{b}}$

8- E. coli $W T+\left[\mathrm{P}_{\text {van }}-n / p /\right]$

C

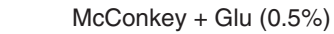

d McConkey + Glu (0.5\%)
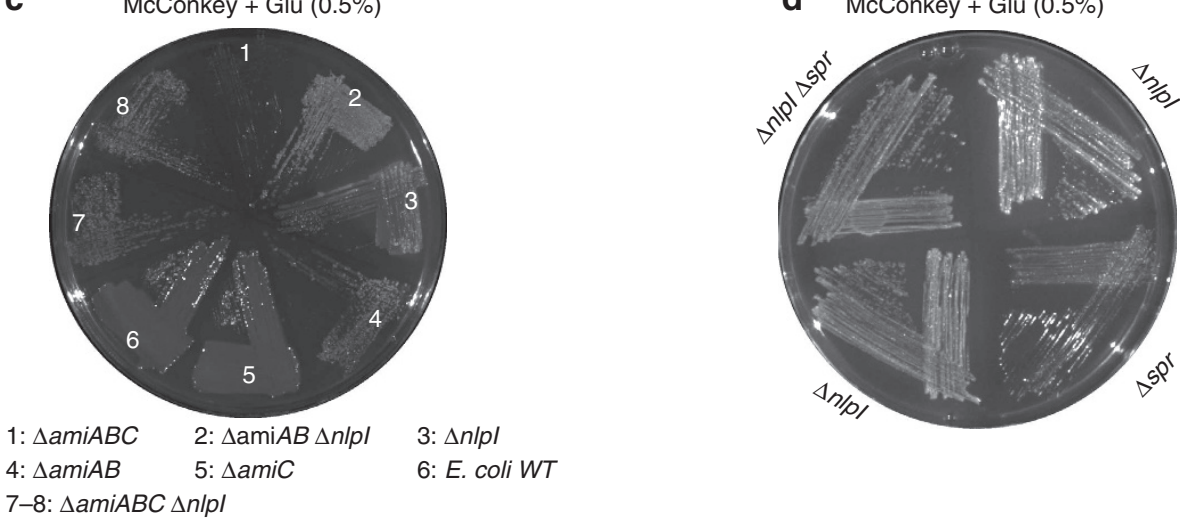

4: $\triangle$ amiAB 5 : $\triangle$ :

Figure 5 | Amidase and endopeptidase control LPS-dependent barrier function in E. coli. (a) Effect of nlpl loss on growth on McConkey agar supplemented with $0.5 \%$ glucose (McCG) in WT and $\triangle A B C$ strains. Nlpl expression in $\triangle A B C$ nIp/W24STOP prevents growth on McCG. Note that the presence of the vector appears to compromise growth of the n/p/ mutant. (b) Representative DIC micrographs of $\triangle A B C$ I $/$ / W24STOP harbouring the empty vector and a derivative carrying $\mathrm{nlpl}$. Scale bar, $4 \mu \mathrm{m}$. (c) $n / p l$-dependent effect on McCG growth in different amidases mutant backgrounds, relative to $W T$ and $\triangle A B C$. Deletion of $n / p l$ in the $\triangle A B C$ mutant restores the ability to grow on McCG on the indicated strains. (d) Deletion of $n / p l$ in $\triangle$ spr cells restores the ability to grow on $M C C G$ agar plates on the indicated strains.

and DD-endopeptidases in E. coli, we searched for putative DDendopeptidases encoded in the $W$. chondrophila genome using E. coli Spr, YdhO and YebA as BLASTP queries. After considering the genomic context where the candidates are encoded, we focused our attention on the gene annotated as $n l_{p} D\left(n l_{p} D^{W c h}\right)$. $n l p D^{W c h}$ is embedded within a cluster of genes predicted to code for cell wall and division functions (Fig. 1c). Although it is difficult to predict from the primary structure if the $\mathrm{NlpD}^{W c h}$ translation product has endopeptidase activity, the presence of two putative LysM domains (Fig. 1d; Supplementary Fig. 6) that are known to mediate PG-binding and/or septal localization in other proteins ${ }^{32}$ along with a $36 \%$ similarity with $\mathrm{NlpD}^{E c o}$ (Supplementary Fig. 6) make it a strong candidate to act in remodelling of a PG-like polymer and/or chlamydial division.

Having shown that the E. coli $\Delta n l p I:: \mathrm{cm}^{R}$ cells are a suitable background in which to probe for endopeptidase activity, we then tested whether expression of $\mathrm{NlpD}^{W c h}$ reduces the plating efficiency of $\Delta n l p I:: \mathrm{cm}^{R}$ cells, akin to expression of Spr or YdhO, without affecting $W T$ cells (Fig. 6a). Indeed, $W T$ NlpD ${ }^{W c h}$ but not mutant variants harbouring missense mutations in conserved residues within the LysM domain (Supplementary Fig. 6) caused a strong reduction in plating efficiency of $\Delta n l p I:: \mathrm{cm}^{R}$ cells compared with WT cells (Supplementary Fig. 7A). Several of these missense mutants accumulated NlpD ${ }^{W c h}$ to similar levels as $W T$ (Supplementary Fig. 7B), suggesting that conserved residues in the LysM domain are required for function. A similar reduction in plating efficiency was observed upon expression of the NlpD orthologue from Simkania negevensis or Parachlamydia acanthamoebae (NlpD ${ }^{S n e}$ or $\mathrm{NlpD}^{P a c}$, respectively) in $\Delta n l p I:: \mathrm{cm}^{R}$ cells, but not in WT E. coli (Fig. 6b). Moreover, induction of $\mathrm{NlpD}^{W c h}, \mathrm{NlpD}^{\text {Sne }}$ or $\mathrm{NlpD}^{P a c}$ expression caused an efficient release of LacZ from E. coli $\Delta n l p I:: \mathrm{cm}^{R}$ cells and only poorly from $W T$ E. coli cells (Fig. 6c). To confirm that NlpD ${ }^{W c h}$ can affect $E$. coli PG, we conducted HPLC analysis of muropeptides released from sacculi of $\triangle A B C$ cells expressing $\mathrm{NlpD}^{W c h}$. This revealed a similar increase in muro-tetrapeptide monomer to dimer ratio (Table 1; Supplementary Fig. 5A,B) compared with the empty vector, as that resulting from the loss of NlpI. Interestingly, the increase in tetrapeptide monomer to dimer ratio was mitigated upon co-expression of $\mathrm{AmiA}^{W c h}$ with $\mathrm{NlpD}^{\text {Wch }}$ (Table 1; Supplementary Fig. 5), despite near-identical steady-state levels of $\mathrm{NlpD}^{W c h}$ in cells with the $\mathrm{AmiA}^{W c h}-\mathrm{NlpD}^{W c h}$ co-expression compared with cells with the $\mathrm{NlpD}^{W c h}$ single expression plasmid (Supplementary Fig. 2C), suggesting that AmiA ${ }^{W c h}$ is epistatic over NlpD ${ }^{W c h}$ and, thus, that they act in the same pathway.

Next, we explored the expression and localization of NlpD ${ }^{W c h}$ in W. chondrophila grown in Vero cells. RT-PCR (Fig. 4a) and immunoblotting using polyclonal antibodies to $\mathrm{NlpD}^{\text {Wch }}$ (Supplementary Fig. 4) showed that NlpD ${ }^{W c h}$ is indeed expressed. Importantly, IFM performed on Vero cells $24 \mathrm{~h}$ p.i. with W. chondrophila revealed fluorescent bands of $\mathrm{NlpD}^{\text {Wch }}$ at midcell in $50.8 \pm 1.1 \%$ of constricted cells (Fig. $4 \mathrm{~b}$ ) and it can already be seen at the septum early during constriction (in $47 \%$ of the cells, Supplementary Fig. 8), with a significant increase in frequency at the later stages of constriction. Quantitative analysis of the fluorescence traces from NlpD ${ }^{W c h}$, DAPI $\left(4^{\prime}, 6\right.$-diamidino2-phenylindole)-stained chromosome and the anti-Waddliastained cell envelope revealed a sharp increase in $\mathrm{NlpD}^{\text {Wch }}$ abundance at the medial site, in between two broad peaks of 

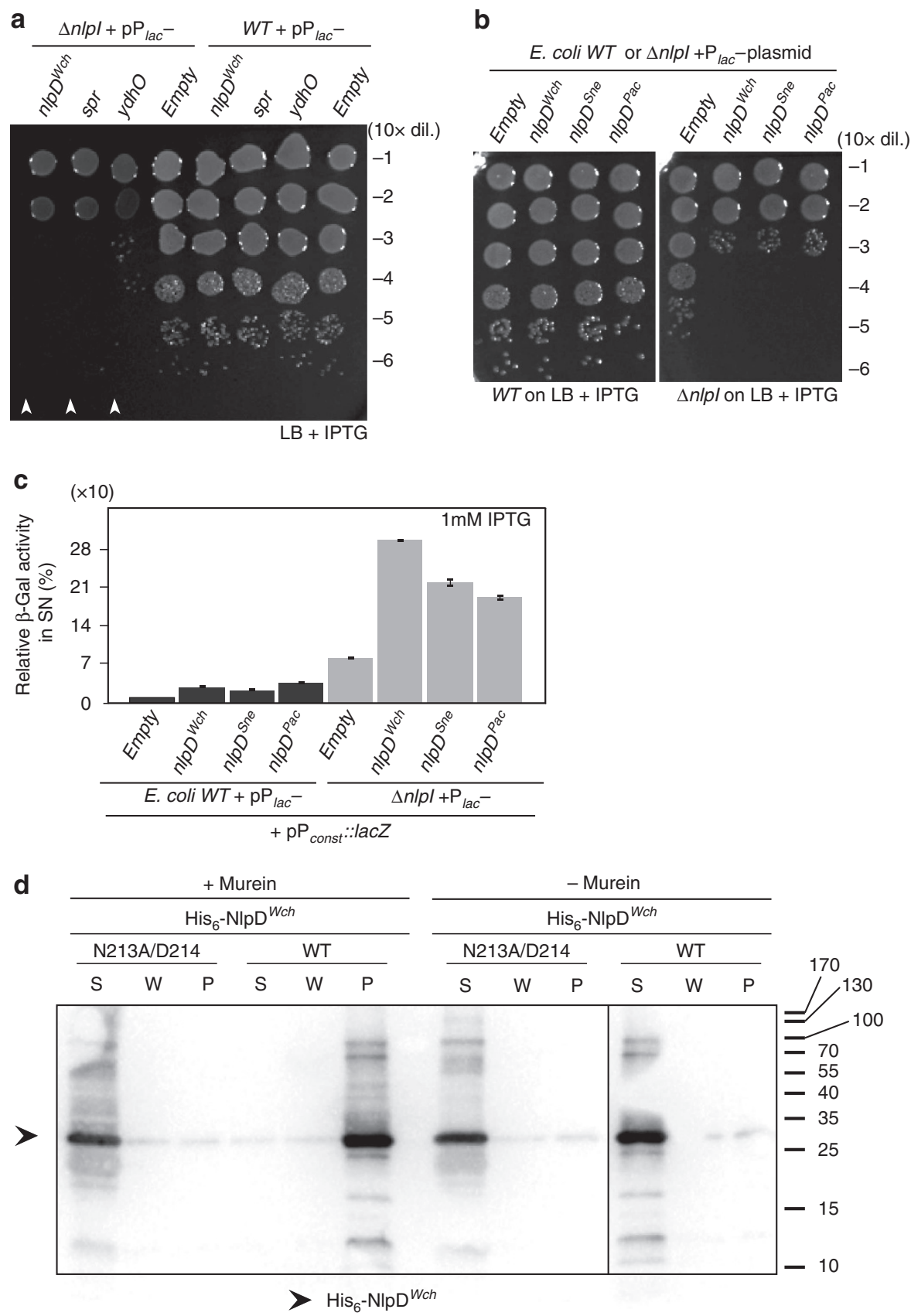

Figure 6 | Phenotypic and biochemical characterization of NIpD ${ }^{\text {Wch }}$. (a) Effect of overexpression of NlpD Wch from pTrc99a (vector) phenocopies the effect of Spr and YdhO overproduction on plating efficiency of E. coli WT and $\Delta n / p /$ cells. Shown is a dilution series of the indicated strains. (b) Effect of overexpression of $\mathrm{NlpD}^{W c h}, \mathrm{NlpD}^{\text {Sne }}$ or $\mathrm{NlpD}^{\text {Pac }}$ from $\mathrm{P}_{\text {lac }}$ on pSRK on plating efficiency of WT and $\Delta$ nlpl E. coli cells. Shown is a dilution series of cells carrying pSRK (vector) derivatives expressing NIpD Wch from $P_{\text {lac }}$ plated on LB with or without inducer ( $1 \mathrm{mM} \mathrm{IPTG)} \mathrm{of} \mathrm{the} \mathrm{indicated} \mathrm{strains.} \mathrm{(c)} \mathrm{Lytic} \mathrm{activity}$ of $\mathrm{NlpD}^{W c h}, \mathrm{NlpD}^{\text {Sne }}$ or $\mathrm{NlpD}^{\text {Pac }}$ expressed from $\mathrm{P}_{\text {lac }}$ on $\mathrm{pSRK}$ in WT and $\Delta$ nlpl E. coli cells carrying the LacZ-expressing plasmid pLac290-P $\mathrm{P}_{\text {const }}:$ Iac (used because the E. coli parent, TB28, is lacZ minus). Beta-galactosidase activities were measured on SN of induced and non-induced cultures of WT E. coli carrying $\mathrm{P}_{\mathrm{lac}}-\mathrm{nl} \mathrm{pD}$ constructs. Error bars show the s.d. Data are from three biological replicates. (d) Binding of different His ${ }_{6}-\mathrm{NlpD}^{\text {Wch }}$ variants to E. coli murein (PG) sacculi. Purified WT and mutant NIpDWch ( $3 \mu \mathrm{g}$ each) were incubated with or without $1 \mathrm{mg}$ of E. coli sacculi. Sacculi were pelleted by ultracentrifugation and washed once with buffer. Immunoblotting with antibodies to NlpD Wch was used to reveal NIpD Wch in the supernatant (S), the wash fraction $(\mathrm{W})$ or the pellet $(\mathrm{P})$ fraction. The size markers (in $\mathrm{kDa}$ ) are indicated on the right. The arrow on the left denotes the position of $\mathrm{His}_{6}-\mathrm{NlpD}$ Wch.

DAPI-stained DNA flanked by the cell envelope (Fig. 4c). This septal localization is still maintained for 4 hours after inhibition of division with penicillin, an inhibitor of PG transpeptidation enzymes $(\mathrm{Pbp} 2 / 3)$, or with phosphomycin, an inhibitor of the lipid II biosynthetic enzyme MurA (Fig. 4b). However, $20 \mathrm{~h}$ later only peripheral $\mathrm{NlpD}^{W c h}$ was observed under both conditions (septal only in $4.4 \%$ and $2.4 \%$ of dividing cells, respectively; Fig. 4d and Supplementary Fig. 8). By contrast, the early cell division marker RodZ $\mathrm{Z}^{W c h}$ was still septal in the presence of the transpeptidation inhibitor (septal in $43.4 \%$ of dividing cells), but not when lipid II biosynthesis is blocked (Fig. 4d), as reported recently ${ }^{11}$. 


\section{Table 1 | Changes in muropeptides in various $E$. coli strains.}

\begin{tabular}{|c|c|c|}
\hline Strain & Tri:Tetra (a:c) ${ }^{\star}$ & Tetra:(Tetra) $)_{2}$ (c:d) ${ }^{\dagger}$ \\
\hline WT & $33 \pm 2.79$ & $146 \pm 2.02$ \\
\hline$\triangle A B C$ & $27 \pm 3.63$ & $165 \pm 2.10$ \\
\hline$\Delta n l p l:: K m$ & $44 \pm 2.17$ & $203 \pm 4.67$ \\
\hline$\triangle A B C \mathrm{nlpl}^{\mathrm{W} 24 S T O P}$ & $40 \pm 4.79$ & $263 \pm 2.89$ \\
\hline$\triangle A B C+$ plasmid & $33 \pm 3.20$ & $148 \pm 3.39$ \\
\hline$\triangle A B C+P_{\text {yan }}:: a m i A^{W c h}$ & $24 \pm 1.77$ & $140 \pm 5.44$ \\
\hline$\triangle A B C+P_{\text {van }}:: n / p D^{W c h}$ & $30 \pm 2.86$ & $291 \pm 3.94$ \\
\hline$\triangle A B C+P_{\text {van }}:[a m i A-n l p D]^{W c h}$ & $21 \pm 4.82$ & $146 \pm 5.37$ \\
\hline
\end{tabular}

Murein (sacculi) was extracted from E. coli strains, digested with mutanolysin, analysed by HPLC and muropeptide ratios calculated from the HPLC chromatograms (peaks a:c, c:d) shown in Supplementary Fig. 5 (see Methods). Error is shown as the s.d. HPLC runs were performed in triplicate.

${ }^{\star}$ Ratio between tri-peptide and tetra-peptide.

tRatio between tetra-peptide and tetra-peptide dimers.

As these findings suggest that chlamydial NlpD is recruited to the division septum by its substrate, a PG-like D-amino acidcontaining peptide polymer, we used a pelleting assay with intact and purified E. coli polymeric PG (sacculi) to determine whether purified $W T$ or mutant $\mathrm{His}_{6}$-tagged NlpD ${ }^{W c h}\left(\mathrm{His}_{6}-\mathrm{NlpD}^{W c h}\right)$ can indeed bind polymeric PG in vitro (Fig. 6d). E. coli sacculi pulled down WT $\mathrm{His}_{6}-\mathrm{NlpD}^{W c h}$, but not mutant derivative lacking two conserved residues in the LysM domain (N213A/ D214A; Supplementary Fig. 6), indicating that $\mathrm{NlpD}^{W c h}$ can bind PG directly.

\section{Discussion}

Our data support a model in which $\mathrm{NlpD}{ }^{W c h}$ recognizes a PGlike polymer at the division septum, while AmiA trims this polymer or lipid II molecules throughout the envelope and possibly at the division furrow in the final stages of division, perhaps to lower NOD1/2-inducing MurNAc-peptide signals ${ }^{12,33}$ during chlamydial infections, akin to the staphylococcal autolysins that prevent detection by the Drosophila innate immune system ${ }^{33}$. Interestingly, a PG-like polymer was recently extracted from the 'environmental' chlamydia Protochlamydia amoebophila, an amoebal symbiont, but similar attempts were unsuccessful for Simkania negevensis ${ }^{34}$. Nevertheless, we found that the $S$. negevensis genome encodes functional AmiA and $\mathrm{NlpD}\left(\mathrm{AmiA}^{\text {Sne }}\right.$ and $\mathrm{NlpD}^{\text {Sne }}$ ) that are active on E. coli PG, suggesting that PG-like material is also present in S. negevensis. It is possible that pervasive PG synthesis throughout the envelope (giving rise to intact sacculi) is a feature of chlamydial lineages that establish symbiotic relationships with amoebae, while chlamydial human pathogens only produce a cryptic, modified, short-lived, thin and/or spatially restricted PG. In light of the recent evidence that $\mathrm{PG}$ synthesis can indeed occur de novo (that is, in the absence of a preexisting template) in Bacillus subtilis cells $^{35}$, it is conceivable that a PG-like structure is confined temporally and spatially to the division septum in chlamydial pathogens. Recent experiments using fluorescently labelled D-amino-dipeptides provided evidence of a septal peptide component in PG (or in lipid II) of Chlamydia trachomatis ${ }^{10}$, in support of the earlier discovery of the SEP antigen (recognized by antibodies raised against the mycobacterial cell wall containing RIBI adjuvant) at the division septum of $C$. trachomatis and Chlamydia psittaci ${ }^{9}$. Our data indicate that a septal PG-like polymer in the human chlamydial pathogen $W$. chondrophila is both a substrate and an important localization cue for a septal PG-binding protein (NlpD) during chlamydial cell division. Moreover, using purified components Klöckner et al. ${ }^{26}$ provide compelling biochemical evidence that $C$. pneumoniae NlpD has carboxypeptidase activity in vitro.
The notion that human chlamydial pathogens rely on a PG-like peptide polymer at the division site and that they localize NlpD to this site in the absence of an FtsZ homologue raises the important evolutionary question how a PG-like division septum is positioned in different bacteria. Our recent identification of the RodZ homologue RodZ $Z^{W c h}$ as an early septal protein ${ }^{11}$ along with the finding reported here that $\operatorname{Rod} Z^{W c h}$ is still septal under conditions when $\mathrm{NlpD}^{W c h}$ is dispersed (in the presence of penicillin) suggest that $\operatorname{Rod} Z^{W c h}$ localizes to the septum before $\mathrm{NlpD}^{W c h}$ and that it could play a key role in orchestrating septal assembly (via PBPs) and dissolution (via NlpD ${ }^{W c h}$ and possibly AmiA $\left.{ }^{W c h}\right)$. While FtsZ is known to be dispensable in another bacterial phylum (the Firmicutes) when PG is absent ${ }^{13,14}$, the only known case of FtsZ-independent PG-based septation has been described for filamentous bacteria from the phylum Actinobacteria (genus Streptomyces) that rely on FtsZ exclusively for septation during spore development, while crosswalls formed during vegetative (hyphal) growth do not require Fts $\mathrm{Z}^{36}$. As the Streptomyces do not encode an obvious RodZ orthologue in their genomes, different solutions have emerged for the synthesis of a PG-based septum and the subsequent recruitment of remodelling enzymes in diverse bacterial phyla and even in eukaryotic organelles ${ }^{1,37}$.

\section{Methods}

Bacterial strains and growth conditions. Strains and plasmids used in this study are listed in Supplementary Table 2 and their constructions are described in the Supplementary Methods section. E. coli strains were grown at $30^{\circ} \mathrm{C}$ in LuriaBertani (LB) broth, LB-agar ${ }^{38}$ or McConkey agar supplemented with gentamycin $\left(10 \mu \mathrm{g} \mathrm{ml}^{-1}\right)$, IPTG (isopropyl- $\beta$-thio-galactopiranoside, $1 \mathrm{mM}$ ), as needed or otherwise indicated. C. crescentus strains were grown at $30^{\circ} \mathrm{C}$ in peptone yeast extract supplemented with gentamycin $\left(1 \mu \mathrm{g} \mathrm{ml}^{-1}\right)$ as needed. Plasmids were introduced in E. coli by electroporation, chemical transformation or conjugation. W. chondrophila ATCC VR- $1470^{\mathrm{T}}$ was grown in Vero cells as previously described ${ }^{5}$. Overnight cell cultures containing originally $10^{5}$ cells $^{-1}$ were infected with a 2,000 $\times$ dilution of $W$. chondrophila. The cells were then centrifuged for $15 \mathrm{~min}$ (to improve contact of $W$. chondrophila) at $1,790 \mathrm{~g}$, incubated $15 \mathrm{~min}$ at $37^{\circ} \mathrm{C}$ and washed with PBS before addition of fresh media.

Differential interference contrast microscopy. Cultures were grown at $30^{\circ} \mathrm{C}$ in LB medium unless otherwise indicated. Unless otherwise indicated cells were imaged by DIC (Differential Interference Contrast) optics on microscope slides harbouring a thin (1\%) agarose pad. A Zeiss Axioplan 2 microscope fitted with an HQ Snapshot camera, a Zeiss oil immersion objective $(\times 100 / 1.45$ numerical aperture) were used to acquire DIC images using software from Metamorph (Universal Imaging). Cells and ghosts were quantified using ImageJ software (http://rsbweb.nih.gov/ij/).

Immunofluorescence and confocal microscopy. Infected Vero cells on coverslips were fixed with ice-cold methanol for $5 \mathrm{~min}$ at room temperature. Infection rate inclusions and aberrant bodies were quantified by fluorescence microscopy by counting a minimum of hundred cells in duplicate ${ }^{5}$. Images were taken by confocal microscopy using a Zeiss LSM 510 Meta (Zeiss, Oberkochen, Germany). Images were then treated and quantified using ImageJ software.

AmiA and NIpD purification and production of antibodies. $\mathrm{His}_{6}-\mathrm{NlpD}^{W c h}$ protein and the N213A/D214A mutant derivative were expressed from pET28a in E. coli Rosetta (DE3)/pLysS (Novagen, Madison, WI) and purified under native conditions using $\mathrm{Ni}^{2}+$ chelate chromatography. A $5 \mathrm{ml}$ overnight culture was diluted into 11 of pre-warmed LB. OD $600 \mathrm{~nm}$ were monitored until $\mathrm{OD}_{600 \mathrm{~nm}}=$ $\sim 0.3-0.4$, then $1 \mathrm{mM}$ IPTG was added to the culture and growth continued. After $3 \mathrm{~h}$ cells were pelleted, and resuspended in $25 \mathrm{ml}$ of lysis buffer $(10 \mathrm{mM}$ Tris $\mathrm{HCl}$ ( $\mathrm{pH} 8$ ), $0.1 \mathrm{M} \mathrm{NaCl}, 1.0 \mathrm{mM} \beta$-mercaptoethanol, $5 \%$ glycerol, $0.5 \mathrm{mM}$ imidazole Triton X-100 0.02\%). Cells were sonicated (Sonifier Cell Disruptor B-30; Branson Sonic Power. Co., Danbury, CT) on ice using 12 bursts of $20 \mathrm{~s}$ at output level 5.5 After centrifugation at $4,300 \mathrm{~g}$ for $20 \mathrm{~min}$, the supernatant was loaded onto a column containing $5 \mathrm{ml}$ of Ni-NTA agarose resin pre-equilibrated with lysis buffer Column was rinsed with lysis buffer, $400 \mathrm{mM} \mathrm{NaCl}$ and $10 \mathrm{mM}$ imidazole, both prepared in lysis buffer. Fractions were collected (in $300 \mathrm{mM}$ Imidazole buffer, prepared in lysis buffer) and used to immunize New Zealand white rabbits (Josman LLC, Napa, CA).

$\mathrm{His}_{6}$-SUMO-AmiA ${ }^{W c h}$ was expressed from pCWR547-amiA ${ }^{W c h}$ over in E. coli Rosetta (DE3)/pLysS and purified in denaturing buffer (8 M Urea, $100 \mathrm{mM}$ 
$\mathrm{NaH}_{2} \mathrm{PO}_{4}, 25 \mathrm{mM}$ Tris). A $5 \mathrm{ml}$ overnight culture was diluted into $1 \mathrm{~L}$ of prewarmed LB. $\mathrm{OD}_{600 \mathrm{~nm}}$ was monitored until $\mathrm{OD}_{600 \mathrm{~nm}}=\sim 0.3-0.4$, then $1 \mathrm{mM}$ IPTG was added to the culture transferred at room temperature for $5 \mathrm{~h}$. Thereafter, cells were pelleted, resuspended in $25 \mathrm{ml}$ of lysis buffer $(10 \mathrm{mM}$ Tris $\mathrm{HCl}(\mathrm{pH} 8), 0.1 \mathrm{M}$ $\mathrm{NaCl}, 1.0 \mathrm{mM} \beta$-mercaptoethanol, $5 \%$ glycerol, $0.5 \mathrm{mM}$ imidazole, Triton X-100 $0.02 \%$ ). Cells were sonicated (Sonifier Cell Disruptor B-30; Branson Sonic Power. Co., Danbury, CT) on ice using 12 bursts of $20 \mathrm{~s}$ at output level 5.5. After centrifugation at $4,300 \mathrm{~g}$ for $20 \mathrm{~min}$, the supernatant were discarded and the pellet resuspended in $25 \mathrm{ml}$ of Buffer B (denaturing buffer, $\mathrm{pH} \mathrm{8.0),} \mathrm{then} \mathrm{centrifuged} \mathrm{at}$ $4,300 \mathrm{~g}$ for $20 \mathrm{~min}$, the supernatant was loaded onto a column containing $5 \mathrm{ml}$ of Ni-NTA agarose resin. Column was rinsed with Buffer B, Buffer C (denaturing buffer, $\mathrm{pH}$ 6.3) and eluted with Buffer $\mathrm{E}$ (denaturing buffer, $\mathrm{pH}$ 4.5). Fractions were collected, the protein was excised from a 15\% SDS polyacrylamide gel and used to immunize New Zealand white rabbits (Josman LLC, Napa, CA).

Immunoblots. Pelleted cells were resuspended in $1 \times$ SDS sample buffer $(50 \mathrm{mM}$ Tris- $\mathrm{HCl}$ (pH 6.8), $2 \%$ SDS, $10 \%$ glycerol, $1 \% \beta$-mercaptoethanol, $12.5 \mathrm{mM}$ EDTA, $0.02 \%$ Bromophenol Blue), heated to $95^{\circ} \mathrm{C}$ for $10 \mathrm{~min}$ and stored at $-20^{\circ} \mathrm{C}$. The resulting cell extracts were resolved on SDS-PAGE gels and transferred onto PVDF (polyvinylidenfluoride) membranes.

PVDF membranes (Merck Millipore Headquarters, Billerica, MA) were blocked with TBS, $0.05 \%$ Tween 20 and $5 \%$ dry milk for $1 \mathrm{~h}$ and then incubated for $1 \mathrm{~h}$ with the primary antibodies diluted in TBS, $0.05 \%$ Tween 20 and 5\% dry milk. The different antisera (custom produced, as described above) were used at the following dilutions: anti-AmiA ${ }^{W c h}(1: 10,000)$, anti-NlpD ${ }^{W c h}(1: 10,000)$. The membranes were washed four times for $5 \mathrm{~min}$ in TBS and incubated $1 \mathrm{~h}$ with the secondary antibody (HRP-anti-Rabbit 1:10,000) diluted in TBS, $0.05 \%$ Tween 20 and $5 \%$ dry milk. The membranes were finally washed again four times for $5 \mathrm{~min}$ in TBS and revealed with Immobilon Western Blotting Chemoluminescence HRP substrate (Merck Millipore Headquarters, Billerica, MA).

Phage manipulation, lysozyme and antibiotic sensitivity tests. The bacterial strains used in the present study were used to produce $\Phi P 1$ lysates and tested for phage P1 sensitivity ${ }^{38}$. Saturated cultures of E. coli TB28 ( $\operatorname{lacZYA}<>$ frt $)$ were used to produce $\Phi P 1$ lysates. Cells from overnight cultures were diluted 1:100 in LB with $25 \mathrm{mM} \mathrm{CaCl} 2$ and infected with ФP1 lysate. Phage titres were calculated by spot and plating methods. Briefly $1.5 \mathrm{ml}$ of cells from overnight cultures were pelleted and resuspended in $0.3 \mathrm{ml}$ of $\mathrm{LB}$ with $5 \mathrm{mM}^{\text {of }} \mathrm{CaCl}_{2}$, and then incubated for $10 \mathrm{~min}$ at $37^{\circ} \mathrm{C}$. A total of $0.05 \mathrm{ml}$ of $\Phi P 1$ were added to the suspension and incubated at $37^{\circ} \mathrm{C}$ for $20 \mathrm{~min}$. Serial dilution of the suspension were made and added to $4 \mathrm{ml}$ of LB top agar supplemented with $5 \mathrm{mM} \mathrm{CaCl}_{2}$, plates were then incubated overnight at $37^{\circ} \mathrm{C}$ or until plaques are visible. The titre of the phage was calculated as plaque-forming units (pfu) per $\mathrm{ml}$, then percentage of the titre relative to WT E. coli was calculated and reported as the $\log _{10}$ of the percentage. Values reported in the figures come from three independent biological replicas.

Sensitivity of bacterial strains presented in this study were determined as follows: $5 \mathrm{mg}$ of lysozyme (AppliChem) and bacitracin (Sigma), respectively, were spotted on LB top agar containing $0.250 \mathrm{ml}$ of saturated overnight cultures of the strains to be tested and incubated overnight at $30^{\circ} \mathrm{C}$. Diameter of the inhibition halo was measured from three biological replicates.

Muropeptide analysis. Chemical composition of PG was determined by the standardized procedure developed by Cecolabs (Tübingen, Germany). For PG isolation and characterization the strains were grown overnight in LB or peptone yeast extract media unless otherwise indicated. Cells were harvested by centrifugation $\left(4,300 \mathrm{~g}, 20 \mathrm{~min}\right.$ at $\left.4{ }^{\circ} \mathrm{C}\right)$, supernatant was discarded and cells resuspended in $20 \mathrm{ml}$ of phosphate buffered saline (pH 7.4; 1x PBS) and then boiled for $15 \mathrm{~min}$, the resulting extract was centrifuged and after discarding the supernatant the pellet was stored at $4{ }^{\circ} \mathrm{C}$.

Briefly, cell pellets were resuspended and boiled in SDS, cell wall material was harvested by centrifugation and broken with glass beads. Broken cell wall was digested with mutanolysin and analysed by HPLC ${ }^{39}$. HPLC analyses were performed with an Agilent 1200 system with a Prontosil C18-RP column (Bischoff Chromatography, Leonberg, Germany) ${ }^{40}$. Selected peaks were identified by matrixassisted laser desorption ionization and electrospray mass spectrometry.

Quantitative analysis of selected peaks was done by integration of the peak area using the trapezoidal rule. The area of each peak was then used to derive the ratios of cell wall components among the different strains.

Murein (sacculi) pull-down assay. $\mathrm{NlpD}^{W c h}-\mathrm{His}_{6}$ and $\mathrm{NlpD}^{W c h}{ }_{\mathrm{N} 213 \mathrm{~A} \mathrm{D} 214 \mathrm{~A}}-\mathrm{His}_{6}$ were overproduced in E. coli Rosetta $(\lambda \mathrm{DE} 3) / \mathrm{pLys}$. Proteins were purified by nickel affinity chromatography as described above, concentrated by ultrafiltration in Amicon $3 \mathrm{~K}$ columns (Millipore) and stored at $-80^{\circ} \mathrm{C}$ in Binding buffer $(20 \mathrm{mM}$ Tris- $\mathrm{HCl}, 1 \mathrm{mM} \mathrm{MgCl}_{2} 30 \mathrm{mM} \mathrm{NaCl}, 0.05 \%$ Triton X-100, pH 6.8) containing $50 \%$ glycerol. Bradford assay was used to determine the protein concentration in each sample. E. coli murein (sacculi) was purchased from Cecolabs (Tuebingen, Germany) and resuspended in binding buffer at a concentration of $10 \mathrm{mg} \mathrm{ml}^{-1}$. Proteins $(3 \mu \mathrm{g}$ or $6 \mu \mathrm{g})$ were added to $1 \mathrm{mg}$ murein in a total volume of $100 \mu \mathrm{l}$ and incubated on ice for $30 \mathrm{~min}$. Murein from samples was collected by centrifugation in a Beckman SW55Ti rotor at $303,648 \mathrm{~g}$ for $30 \mathrm{~min}$ at $4{ }^{\circ} \mathrm{C}$. Sedimented murein was resuspended in $0.1 \mathrm{ml}$ of cold binding buffer and centrifuged again. Murein pellets were resupended in $0.02 \mathrm{ml}$ of cold binding buffer. The supernatant of the first centrifugation step (S), the supernatant of the washing step (W) and the pellet (P) were analysed by SDS-PAGE followed by immunoblot with anti-NlpD antiserum (see immunoblots for details).

Assay to determine the lytic activity of chlamydial amidases. $W T$ and $\triangle A B C$ E. coli cells harbouring pSRK $\left(\mathrm{P}_{\text {lac }}\right)$ and pMT335 $\left(\mathrm{P}_{\text {van }}\right)$ amidase plasmids were transformed with the low copy plasmid pLac290 harbouring a promoter of $C$.

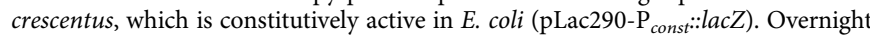
cultures were diluted in fresh $\mathrm{LB}$ and incubated at $30^{\circ} \mathrm{C}$ for $6 \mathrm{~h}$. Culture supernatants (SN) were collected by centrifugation at $20,000 \mathrm{~g}$ for $5 \mathrm{~min}$ at room temperature. SN were diluted 1:10 in $1 \mathrm{ml}$ of $\mathrm{LB}$ and $0.2 \mathrm{ml}$ of diluted SN were used for standard $\beta$-Galactosidase (LacZ) assay ${ }^{38}$.

Quantitative real-time PCR. Infection of Vero cells by W. chondrophila was quantified by quantitative $\mathrm{PCR}^{11}$. At different time points after infection, infected cells were resuspended by scratching. Genomic DNA was extracted from $50 \mu \mathrm{l}$ of cell suspension using the Wizard SV Genomic DNA purification system (Promega, Madison, WI). Elution was processed with $200 \mu \mathrm{l}$ of water. Quantitative PCR was performed using iTaq supermix with ROX (BioRad, Hercules, CA). To detect W. chondrophila, $200 \mathrm{nM}$ of primers WadF4 and WadR4, $100 \mathrm{nM}$ of probe WadS2 and $5 \mu \mathrm{l}$ of DNA were used. Cycling conditions were $3 \mathrm{~min}$ at $95^{\circ} \mathrm{C}$ followed by 40 cycles of $15 \mathrm{~s}$ at $95^{\circ} \mathrm{C}$ and $1 \mathrm{~min}$ at $60^{\circ} \mathrm{C}$ for both PCRs. A StepOne Plus Real-time PCR System (Applied Biosystems, Carlsbad, CA) was used for amplification and detection of the PCR products.

\section{References}

1. Margolin, W. FtsZ and the division of prokaryotic cells and organelles. Nat. Rev. Mol. Cell Biol. 6, 862-871 (2005).

2. Adams, D. W. \& Errington, J. Bacterial cell division: assembly, maintenance and disassembly of the Z ring. Nat. Rev. Microbiol. 7, 642-653 (2009).

3. Uehara, T. \& Bernhardt, T. G. More than just lysins: peptidoglycan hydrolases tailor the cell wall. Curr. Opin. Microbiol. 14, 698-703 (2011).

4. Erickson, H. P., Anderson, D. E. \& Osawa, M. FtsZ in bacterial cytokinesis cytoskeleton and force generator all in one. Microbiol. Mol. Biol. Rev. 74, 504-528 (2010).

5. Bertelli, C. et al. The Waddlia genome: a window into chlamydial biology. PLoS ONE 5, e10890 (2010).

6. Stephens, R. S. et al. Genome sequence of an obligate intracellular pathogen of humans: Chlamydia trachomatis. Science 282, 754-759 (1998).

7. Henrichfreise, B. et al. Functional conservation of the lipid II biosynthesis pathway in the cell wall-less bacteria Chlamydia and Wolbachia: why is lipid II needed? Mol. Microbiol. 73, 913-923 (2009).

8. Ghuysen, J.-m., Goffin, C. \& Inge, C. Lack of cell wall peptidoglycan versus penicillin sensitivity: new insights into the chlamydial anomaly. Antimicrob. Agents Chemother. 43, 2339-2344 (1999).

9. Brown, W. J. \& Rockey, D. D. Identification of an antigen localized to an apparent septum within dividing Chlamydiae. Infect. Immun. 68, 708-715 (2000).

10. Liechti, G. W. et al. A new metabolic cell-wall labelling method reveals peptidoglycan in Chlamydia trachomatis. Nature 506, 507-510 (2014).

11. Jacquier, N., Frandi, A., Pillonel, T., Viollier, P. \& Greub, G. Cell wall precursors are required to organize the chlamydial division septum. Nat. Commun. 5, 3578 (2014).

12. Underhill, D. M. Collaboration between the innate immune receptors dectin-1, TLRs, and Nods. Immun. Rev. 219, 75-87 (2007).

13. Leaver, M., Dominguez-Cuevas, P., Coxhead, J. M., Daniel, R. A. \& Errington, J. Life without a wall or division machine in Bacillus subtilis. Nature 457, 849-853 (2009).

14. Lluch-Senar, M., Querol, E. \& Pinol, J. Cell division in a minimal bacterium in the absence of ftsZ. Mol. Microbiol. 78, 278-289 (2010).

15. Pinho, M. G., Kjos, M. \& Veening, J. W. How to get (a)round: mechanisms controlling growth and division of coccoid bacteria. Nat. Rev. Microbiol. 11, 601-614 (2013).

16. Uehara, T., Parzych, K. R., Dinh, T. \& Bernhardt, T. G. Daughter cell separation is controlled by cytokinetic ring-activated cell wall hydrolysis. EMBO J. 29, 1412-1422 (2010).

17. Ruiz, N., Kahne, D. \& Silhavy, T. J. Transport of lipopolysaccharide across the cell envelope: the long road of discovery. Nat. Rev. Microbiol. 7, 677-683 (2009).

18. Heidrich, C., Ursinus, A., Berger, J., Schwarz, H. \& Höltie, J. V. Effects of multiple deletions of murein hydrolases on viability, septum cleavage, and sensitivity to large toxic molecules in Escherichia coli. J. Bacteriol. 184, 6093-6099 (2002).

19. Baud, D. et al. Waddlia chondrophila: from bovine abortion to human miscarriage. Clin. Infect. Dis. 52, 1469-1471 (2011). 
20. Baud, D. et al. Role of Waddlia chondrophila placental infection in miscarriage. Emerg. Infect. Dis. 20, 460-464 (2014).

21. Yang, D. C. et al. An ATP-binding cassette transporter-like complex governs cell-wall hydrolysis at the bacterial cytokinetic ring. Proc. Natl Acad. Sci. USA 108, E1052-E1060 (2011).

22. Yang, D. C., Tan, K., Joachimiak, A. \& Bernhardt, T. G. A conformational switch controls cell wall-remodelling enzymes required for bacterial cell division. Mol. Microbiol. 85, 768-781 (2012).

23. Greub, G. et al. High throughput sequencing and proteomics to identify immunogenic proteins of a new pathogen: the dirty genome approach. PLoS ONE 4, e8423 (2009).

24. Poindexter, J. S. The caulobacters: ubiquitous unusual bacteria. Microbiol. Rev. 45, 123-179 (1981).

25. Tamaki, S. \& Matsuhashi, M. Increase in sensitivity to antibiotics and lysozyme on deletion of lipopolysaccharides in Escherichia coli strains. J. Bacteriol. 114, 453-454 (1973).

26. Klöckner, A. et al. AmiA is a penicillin target enzyme with dual activity in the intracellular pathogen Chlamydia pneumoniae. Nat. Commun. 5, 4201 (2014).

27. Teng, C. H. et al. NlpI contributes to Escherichia coli K1 strain RS218 interaction with human brain microvascular endothelial cells. Infect. Immun. 78, 3090-3096 (2010).

28. Barnich, N., Bringer, M. A., Claret, L. \& Darfeuille-Michaud, A. Involvement of lipoprotein NlpI in the virulence of adherent invasive Escherichia coli strain LF82 isolated from a patient with Crohn's disease. Infect. Immun. 72, 2484-2493 (2004).

29. Singh, S. K., SaiSree, L., Amrutha, R. N. \& Reddy, M. Three redundant murein endopeptidases catalyse an essential cleavage step in peptidoglycan synthesis of Escherichia coli K12. Mol. Microbiol. 86, 1036-1051 (2012).

30. Hara, H., Abe, N., Nakakouji, M., Nishimura, Y. \& Horiuchi, K. Overproduction of penicillin-binding protein 7 suppresses thermosensitive growth defect at low osmolarity due to an spr mutation of Escherichia coli. Microb. Drug Resist. 2, 63-72 (1996).

31. Tadokoro, A. et al. Interaction of the Escherichia coli lipoprotein NlpI with periplasmic Prc (Tsp) protease. J. Biochem. 135, 185-191 (2004)

32. Buist, G., Steen, A., Kok, J. \& Kuipers, O. P. LysM, a widely distributed protein motif for binding to (peptido)glycans. Mol. Microbiol. 68, 838-847 (2008).

33. Atilano, M. L. et al. Bacterial autolysins trim cell surface peptidoglycan to prevent detection by the Drosophila innate immune system. eLife 3, e02277 (2014).

34. Pilhofer, M. et al. Discovery of chlamydial peptidoglycan reveals bacteria with murein sacculi but without FtsZ. Nat. Commun. 4, 2856 (2013)

35. Kawai, Y., Mercier, R. \& Errington, J. Bacterial cell morphogenesis does not require a preexisting template structure. Curr. Biol. 24, 863-867 (2014).

36. McCormick, J. R. Cell division is dispensable but not irrelevant in Streptomyces. Curr. Opin. Microbiol. 12, 689-698 (2009).
37. Miyagishima, S. Y., Kabeya, Y., Sugita, C., Sugita, M. \& Fujiwara, T. DipM is required for peptidoglycan hydrolysis during chloroplast division. BMC Plant Biol. 14, 57 (2014).

38. Miller, J. H. Experiment in Molecular Genetics (Cold Spring Harbor Laboratory, 1972).

39. de Jonge, B. L., Chang, Y. S., Gage, D. \& Tomasz, A. Peptidoglycan composition of a highly methicillin-resistant Staphylococcus aureus strain. The role of penicillin binding protein 2A. J. Biol. Chem. 267, 11248-11254 (1992).

40. Ute Bertsche, S.-J. Y. et al. Increased cell wall teichoic acid production and D-alanylation are common phenotypes among daptomycin-resistant methicillin-resistant Staphylococcus aureus (MRSA) clinical isolates. PLoS ONE 8, e67398 (2013)

\section{Acknowledgements}

Funding support was from the Fondation Leenaards, the Swiss National Science Foundation (CRSII3_141837) and the Canton de Genève. We thank Tsuyoshi Uehara, Tom Bernhardt, Manjula Reddy and Patrice Moreau for materials; Tsuyoshi Uehara, Tom Bernhardt, Martin Thanbichler and Miguel Valvano for helpful suggestions and Beate Henrichfreise for communicating unpublished results.

\section{Author contributions}

A.F., N.J., G.G. and P.H.V. conceived and designed the experiments. A.F., N.J. and L.T. performed the experiments. A.F., N.J., G.G. and P.H.V. analysed the data. A.F., N.J., G.G. and P.H.V. wrote the paper.

\section{Additional information}

Supplementary Information accompanies this paper at http://www.nature.com/ naturecommunications

Competing financial interests: The authors declare no competing financial interests.

Reprints and permission information is available online at http://npg.nature.com/ reprintsandpermissions/

How to cite this article: Frandi, A. et al. FtsZ-independent septal recruitment and function of cell wall remodelling enzymes in chlamydial pathogens. Nat. Commun. 5:4200 doi: 10.1038/ncomms5200 (2014).

(C) $($ This work is licensed under a Creative Commons Attributioncc. ${ }_{\mathrm{BY}} \mathrm{NC}$ ND NonCommercial-NoDerivs 4.0 International License. The images or other third party material in this article are included in the article's Creative Commons license, unless indicated otherwise in the credit line; if the material is not included under the Creative Commons license, users will need to obtain permission from the license holder to reproduce the material. To view a copy of this license, visit http:// creativecommons.org/licenses/by-nc-nd/4.0/ 